\title{
The number of active palmar sweat glands (palmar sweat index, PSI) as an activation measure in field studies
}

\author{
THOMAS KOHLER, JUTTA DUNKER, and OLAF ZANDER \\ Psychologisches Institut III, Universität Hamburg, Germany
}

\begin{abstract}
The number of active sweat glands within a defined area of a fingertip, called the palmar sweat index (PSI), can be easily determined by means of the plastic fingerprint method. Two experiments were carried out to demonstrate the applicability of this technique in field research and to investigate the properties of the PSI as an activation parameter. In Experiment 1 (in a blood donors' ward), PSI rose significantly during venous puncture and dropped after the cannula had been installed. In Experiment 2 (in a dental office), PSI values increased when patients were seated in the dental chair and rose to a higher level at the dentist's entry. Values after treatment were significantly lower. In both experiments, it was observed that the initial assessments yielded higher PSI values. This is best explained as a reaction to the assessment procedure itself, and it is recommended that the first two prints not be used for the determination of baseline values.
\end{abstract}

Although some studies have been published on the plastic fingerprint technique, which can be used to assess the number of active sweat glands (for a short review, see Köhler \& Vögele, 1989), this method has never received broad attention. This is somewhat surprising, given the simplicity of the technique and its special suitability for field research, considering that attempts to develop other inexpensive and nonintrusive recording apparatus for electrodermal activity outside the laboratory have not been very successful.

The method was first described by Sutarman and Thomson (1952). A drop of polyvinylformal (Formvar) in a solvent is applied to a fingertip. After evaporation of the solvent, a thin plastic layer develops. Since Formvar is repelled by sweat, holes are formed in the film that correspond to open glands and can be counted easily. For evaluation, a defined area around the central finger whorl is used for reference and the resultant number labeled the palmar sweat index (PSI).

The few studies published on the PSI (generally assessed under field conditions) have yielded rather contradictory results. Although some authors reported an increase of values during activation, others observed a decrease (see Köhler \& Vögele, 1989). Some researchers have found no consistent changes and have questioned the validity of the PSI as an indicator of arousal (Weisenberg, Kreindler, Schachat, \& Werboff, 1976). This may be one of the reasons why this method, despite its simplicity, has seldom been used for behavioral assessment.

In previous studies carried out under laboratory conditions, we have found an increase of the PSI during acti-

Correspondence should be addressed to Th. Köhler, Psychologisches Institut III, Universität Hamburg, Von-Melle-Park 5, D-2000 Hamburg 13, Germany. vation (e.g., mental arithmetic, a distressing film) and a decrease thereafter (cf. Köhler \& Troester, 1991; Köhler, Weber, \& Vögele, 1990). Parameters of electrodermal activity changed in the same way, as did (to the extent that they were examined) cardiovascular variables and subjective measures of arousal. We thus conclude that the PSI is a valid activation parameter that is easily assessable in the laboratory.

The purpose of this paper was to demonstrate the applicability of the plastic fingerprint method in field research and to determine whether or not the PSI is a sensitive activation measure. This was done in two experiments. It was hypothesized that PSI values would correspond to the degree of activation during different phases of blood donation and dental treatment.

\section{METHOD}

\section{The Technique of PSI Assessment}

The equipment needed to assess PSI is very simple and inexpensive. A solution of $2 \%-4 \%$ polyvinylformal (Formvar), $1 \%$ dibutylphthalate as a plasticizer, and 1-2-dichlorethan to $100 \%$ as a solvent is often used (also see Köhler et al., 1990). The bottle containing the solution should be open only during application of the drop; it should be properly closed and locked away between investigations.

A drop of this solution is applied to a skin area by means of a glass stopper. Any skin area can be used, depending on the nature of the study. Most suitable-and used in nearly all investigations are the fingerpads, preferably the middle finger of the left hand. The drop should be put around the central whorl, or loop, because this area is easy to find. Exact and consistent localization facilitates standardization within and between the subjects in the same study and also between studies of different laboratories. We advise not changing the reference area within an experiment, since sweat gland activity varies to some extent between different areas of the skin. After the evaporation of the dichlorethan, which usually takes between 15 and $30 \mathrm{sec}$, depending on the amount of the solu- 
tion applied, a plastic layer develops. It can be removed by means of cellophane tape (Scotch Tape) and mounted on a microscope slide or clear acetate sheet.

For evaluation, we used a microscope with $50 \times$ magnification. Since Formvar is repelled by sweat, open (i.e., active) sweat glands appear distinctly as white holes and can easily be counted. Enumeration is carried out by means of a graticule in the ocular corresponding to an area of $2 \times 2 \mathrm{~mm}$ on the plastic layer. Other authors have often evaluated larger areas (e.g., $4 \times 4 \mathrm{~mm}$ ), and it would be preferable for purposes of standardization if the obtained sweat gland counts were reported as counts per $\mathrm{cm}^{2}$.

Since the number and activity of sweat glands may vary across the fingerpad, we always evaluate corresponding areas in a series of prints from the same subject and use a drawing of the finger pattern for the exact identification of the evaluation field. This somewhat painstaking method has the consequence that prints have to be discarded if they are torn in the area chosen for evaluation (for possible ways of overcoming this problem, see Köhler \& Troester, 1991). For evaluation, we have adopted several criteria (see also Köhler et al., 1990). In particular, glands situated in the grooves are not counted; a sweat gland is only considered to be open, hence active, when the inner diameter is at least as great as the diameter of the rim and if the total diameter surpasses a minimum of $50 \mu \mathrm{m}$.

High interrater reliability in PSI determination has been reported by several authors, with coefficients ranging from 0.87 to 0.99 (cf. Johnson \& Dabbs, 1967; Kleinknecht \& Bernstein, 1978; Martens, 1969; Weisenberg et al., 1976). In our studies, interrater reliabilities were calculated by correlating PSI values determined by two raters across all the prints taken from the same person; resultant coefficients were averaged across the subjects of the sample. The values obtained were also satisfactory - for example, a mean of 0.89 in one study (Köhler et al., 1990) and a mean of 0.92 in another (Köhler \& Troester, 1991); the evaluators of the investigations presented here (J.D. and O.Z.) were among the raters of the latter study.

Coefficients of intraindividual parallel test reliability (PSI values from different fingers of the same subjects correlated across points of assessment) had a mean of 0.77 in one study (Köhler et al., 1990), and a mean of 0.83 in another (Köhler \& Troester, 1991). As mentioned above, rather contradictory findings regarding PSI changes have been reported, and the validity of the measure as an activation parameter has been questioned. In all of our studies, however, the PSI rose consistently during activation, changing in a way similar to parameters of electrodermal activity (see introduction).

\section{Subjects and Design}

Experiment 1 was carried out in a blood donors' ward and comprised 30 males between 24 and 64 years old (mean: 45.7 years old). The female experimenter (J.D.) took prints for PSI assessment from the left middle finger at 2-min intervals-four before donation, one at the moment of the venous puncture, three when the cannula was indwelling and blood was taken, and four after removal of the cannula, when the patient was still on the stretcher. Immediately after taking the prints, heart rate (HR), systolic (SBP), and diastolic blood pressure (DBP) were recorded from the left upper arm by means of a semiautomatic sphygmomanometer. Given that the puncture was carried out on the right forearm and that simultaneous taking of the prints and blood pressure measurement on the left arm was expected to cause artifacts, we decided to make the recordings sequentially.

The subjects in Experiment 2 were 12 female and 3 male patients at a dental office. Ages ranged from 18 to 63 years old, with a mean of 39.6 years old. Prints were taken from the right middle fingerthe first four at 2-min intervals in the waiting room before treatment and one after the patient had sat down in the dental chair. The sixth print was taken when the dentist entered and started treatment, the next after treatment when the patient was still seated in the chair, and a final one back in the waiting room. Because the dentist was to the patients' left during treatment, prints had to be taken from the right hand. This time, investigations were carried out by a male experimenter (O.Z.). In order not to disturb the routine of dental treatment, no recordings of other variables were made.

As we carried out only within-subject comparisons and since no differential effects were studied, there was no need to restrict the age of the subjects to a narrow range. However, since there is some reason to assume that PSI values decrease with age (MacKinnon, 1954), this variable should be controlled for between-group comparisons.

\section{Evaluation and Data Reduction}

A few of the prints were torn in the evaluation area; thus, subject counts for repeated measures analyses of variance (ANOVAs) and phase comparisons may be slightly lower than the original sample sizes (see below). Since we had repeatedly observed that the first two PSI values assessed under resting conditions were significantly higher than the following ones (see Köhler \& Troester, 1991; Köhler et al., 1990), we did not use them for baseline determination.

In Experiment 1, the third and fourth recordings of each of the parameters (PSI, SBP, DBP, HR) were averaged to form a baseline value, the three measurements after puncture were averaged to form postpuncture value, and the final measurements after removal of the cannula were averaged to form a follow-up value.

In Experiment 2, the first and second PSI values were again excluded for phase comparisons, and the third and fourth values were averaged to give the baseline value for the waiting room situation before treatment. It was hypothesized that the number of active sweat glands increases when the patient sits down in the dental chair and increases further when the dentist enters and starts the treatment; thereafter, a decrease was expected.

\section{RESULTS}

All hypotheses outlined in the introduction were confirmed. In Experiment 1, a repeated measures one-way ANOVA for PSI over experimental phases (baseline, puncture, postpuncture, and follow-up) yielded a significant effect $[F(3,72)=49.18, p<.001]$. Post hoc $t$ tests revealed that the number of active sweat glands rose significantly from baseline to puncture and fell thereafter to baseline values (see Figure 1). No change as a reaction to the puncture was observed in $\operatorname{SBP}(t=0,75, p=.46$, twotailed), whereas contrary to expectation, DBP and HR fell from baseline to the point of measurement closest to puncture and showed no changes thereafter (see Discussion).

In Experiment 2, a repeated measures ANOVA again yielded significant phase differences $[F(4,44)=17.36$, $p<.001]$. The PSI values increased between sitting in the waiting room and sitting in the dental chair, showed a further significant increase at the dentist's entry, and were considerably lower after completion of treatment (see Figure 2).

\section{DISCUSSION}

Both experiments showed again that the plastic fingerprint method is applicable in field research. In our investigations, it was used without disturbing the routine of blood donation and dental treatment. In addition, the PSI varied consistently across the phases of the investigation, with the highest values attained in the situations we termed "venous puncture" and "being in the dental treatment room." These situations would by common understand- 
ing be considered more stressful or more activating than other situations involved in the investigations (e.g., before and after puncture, outside the treatment room). Although the samples were comparatively small, particularly in Experiment 2 , both activation-related increases and decreases attained statistical significance at the $1 \%$ level of significance or beyond. This is in accordance with some studies (e.g., Early \& Kleinknecht, 1978; Martens, 1969) but at variance with others (see below). In Experiment 2, the measure was able to differentiate significantly between two levels of activation (i.e., expectation of treatment while seated in the dental chair and start of treatment).

In Experiment 1, somewhat surprisingly, SBP did not change consistently during the stages of blood donation, and values in DBP and HR even fell from baseline to puncture. There may be two explanations for this fact. First, it could be that blood pressure and heart rate generally do not change-they may even decrease-as a reaction to venous puncture, whereas other variables, including the PSI, increase. Such a dissociation is not a rare phenomenon, and our finding would certainly not invalidate the PSI as an activation parameter; it may indicate that the PSI is more sensitive and/or less specific as an indicator of psychological states. Second, it should be taken into consideration that recordings of blood pressure and

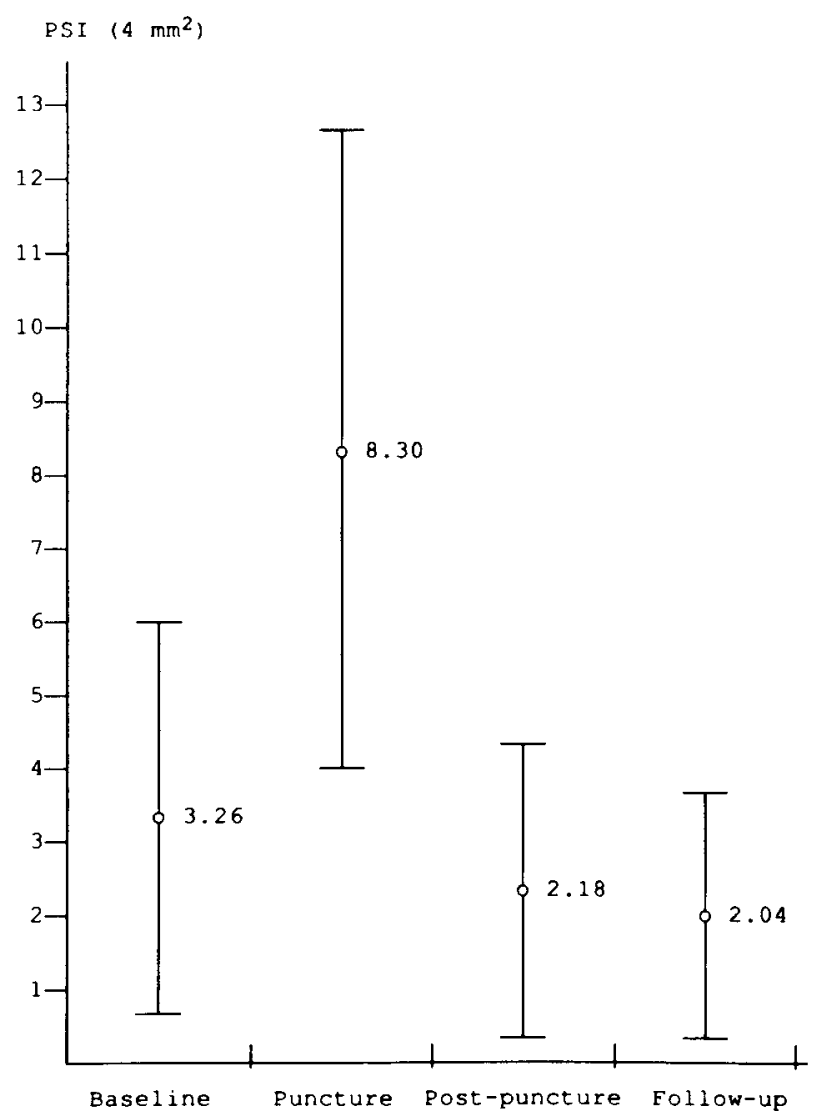

Figure 1. Mean palmar sweat index (PSI) values in several phases of blood donation $(\boldsymbol{N}=26$ ). Differences in PSI between baseline and puncture and between puncture and postpuncture were significant $(p<.001$, two-tailed).

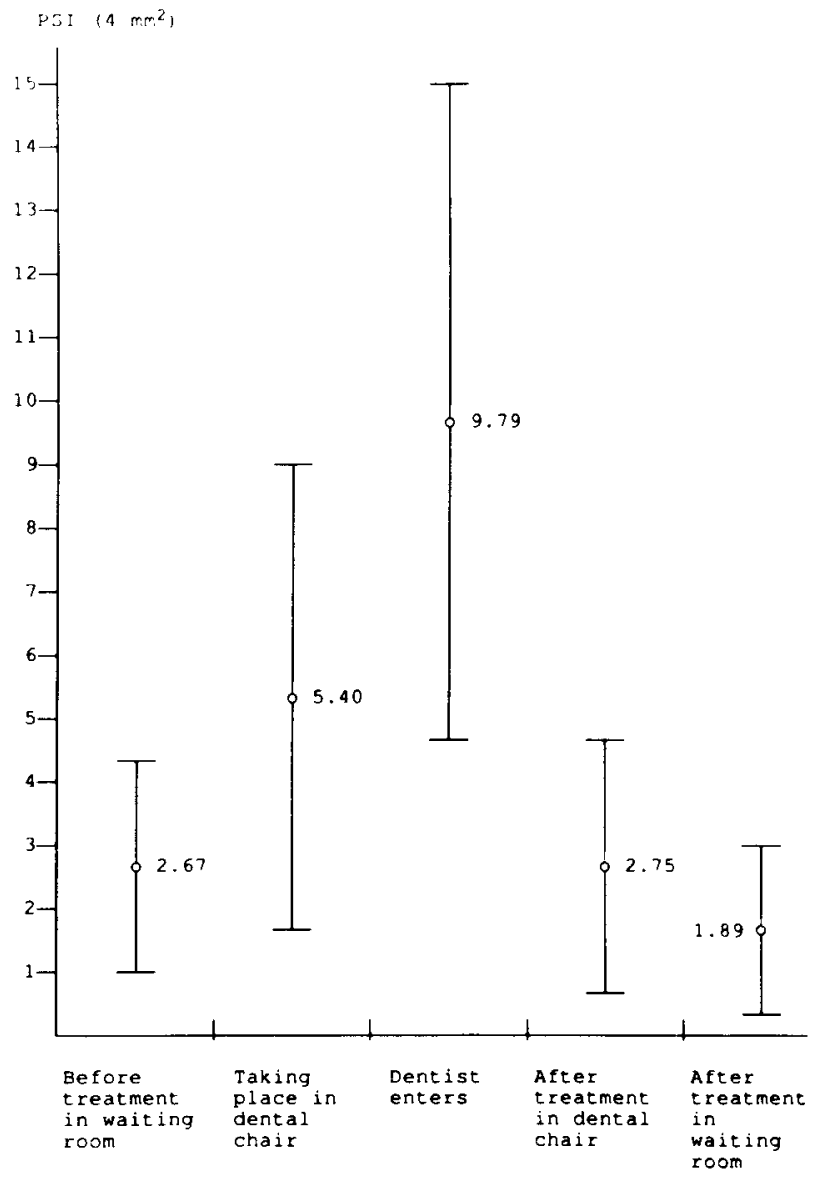

Figure 2. Means of PSI values in several phases of dental treatment $(N=12-15)$. Differences between successive readings were significant $(p<.01$, two-tailed) except for the two after-treatment measures.

heart rate were made after PSI assessment, and thus measurements at this point refer to a moment when the cannula had already been properly installed and the tension had fallen. Concurrent validity of the PSI in the form of a systematic relationship with other measures of activation thus could not be shown in our study. However, this has been done repeatedly in our laboratory under conditions in which synchronization of assessments was easy to realize (see Köhler \& Troester, 1991; Köhler \& Vögele, 1989; Köhler et al., 1990). In the two field studies reported above, criterion validity of the PSI is demonstrated by the result that the highest values of PSI were observed under circumstances commonly considered as activating. Further studies in different situations and with a number of additional parameters should clarify the activating conditions (e.g., physical manipulation, attention-demanding situations, stress-inducing circumstances) under which the PSI changes and should reveal how this is related to changes in other psychophysiological variables.

As mentioned earlier, PSI studies have yielded rather inconsistent results. One finding in our research (see Köhler et al., 1990) casts some light on this problem: We observed that the first two PSI values, obtained under resting 
conditions, were higher than the following ones, probably due to the novelty of the assessment procedure itself. This finding was confirmed in the two experiments presented in this paper: The average of the first two PSI values was higher than the average of the third and fourth values (5.01 \pm 3.65 vs. $3.15 \pm 2.54, p<.001$, in Experiment 1 ; $3.33 \pm 1.64$ vs. $2.67 \pm 1.57, p<.05$, in Experiment 2, both $p s$ one-tailed). In studies in which PSI decreased during stress (e.g., Harrison, 1964, under the threat of an injection) or in which inconsistent changes were observed (e.g., Weisenberg et al., 1976, during dental treatment), researchers had used the initial one or two PSI readings as baseline values. Activation-related increases in PSI may thus have been compensated or overcompensated by a decrease of the number of active sweat glands caused by habituation to the assessment procedure. We therefore recommend taking several additional fingerprints during the initial resting phase and discarding the first two. Under these conditions, the PSI is obviously a valid activation parameter that should be useful in behavioral assessment.

\section{REFERENCES}

Early, C. E., Kleinknecht, R. A. (1978). The Palmar Sweat Index as a function of repression-sensitization and fear of dentistry. Journal of Consulting \& Clinical Psychology, 46, 184-185.
Harrison, J. (1964). The behaviour of the palmar sweat glands in stress. Joumal of Psychosomatic Research, 8, 187-191.

JoHNSON, J. E., DABRS, J. M. (1967). Enumeration of active sweat glands: A simple physiological indicator of psychological changes. Nursing Research, 16, 273-276.

KLEINKNECHT, R., BERNSTEIN, D. A. (1978). The assessment of dental fear. Behavior Therapy, 9, 626-634.

KöHLER, TH. \& TROESTER, U. (1991). Changes of the PSI (palmar sweat index) during mental arithmetic. Biological Psychology, 32, 143-154.

KöHLER, TH., \& VöGElE, C. (1989). Laboratory studies on a potential stress indicator in field research: The Palmar Sweat Index. In D. Siddle $\&$ N. Bond (Eds.), Proceedings of the 24th International Congress of Psychology, Sydney, Australia. Vol. 6. Psychobiology: Issues and Applications (pp. 315-323). Amsterdam: Elsevier

KöHLER, Th., Weber, D. \& VöGELE, C. (1990). The behaviour of the PSI (palmar sweat index) during two stressful laboratory situations. Journal of Psychophysiology, 4, 281-287.

MAcKinnon, P. C. B. (1954). Variations with age in the number of active palmar digital sweat glands. Journal of Neurology, Neurosurgery, \& Psychiatry, 17, 124-126.

MARTENS, R. (1969). Palmar sweating and the presence of an audience. Journal of Experimental \& Social Psychology, 5, 371-374.

Sutarman \& Thomson, M. L. (1952). A new technique for enumerating active sweat glands in man. Journal of Physiology, 117, 51P-52P.

Weisenberg, M., Kreindler, M. L., Schachat, R., Werboff, J. (1976). Interpreting palmar sweat prints: A not-so-simple measure. Journal of Psychosomatic Research, 20, 1-6.

(Manuscript received May 21, 1991; revision accepted for publication June 10, 1992.) 